\title{
USO DI SOSTANZE PSICOATTIVE TRA GLI STUDENTI DI MEDICINA DELL'UNIVERSITÀ FEDERALE DI AMAPÁ NEL 2018
}

\section{ARTICOLO ORIGINALE}

DIAS, Cláudio Alberto Gellis de Mattos ${ }^{1}$, FACCO, Lucas², FECURY, Amanda Alves ${ }^{3}$, MELO, Felipe Rodrigues Martins de ${ }^{4}$, AZEVEDO, Eduardo José Lobato de ${ }^{5}$, RIZZI, Ana Carolina Miranda ${ }^{6}$, OLIVEIRA, Euzébio de ${ }^{7}$, DENDASCK, Carla Viana ${ }^{8}$, SILVA, Anderson Walter Costa $^{9}$, SILVA, Alceu dos Santos ${ }^{10}$, CARDOSO, Rosilene Ferreira ${ }^{11}$, ARAÚJO, Maria Helena Mendonça ${ }^{12}$

DIAS, Cláudio Alberto Gellis de Mattos. Uso di sostanze psicoattive tra gli studenti di medicina dell'Università Federale di Amapá nel 2018. Revista Científica Multidisciplinar Núcleo do Conhecimento. Anno 05, Ed. 05, Vol. 13, pp. 21 31. Maggio 2020. ISSN: 2448-0959, Link di accesso: https://www.nucleodoconhecimento.com.br/saude/discentes-de-medicina, $\quad$ DOI: 10.32749/nucleodoconhecimento.com.br/salute/studenti-di-medicina

\footnotetext{
${ }^{1}$ Biologo, Dottore di Ricerca in Teoria e Comportamento, Professore e ricercatore del Graduate Program in Professional and Technological Education (PROFEPT), Istituto Federale di Amapá (IFAP).

2 Studente del Corso di Medicina dell'Università Federale di Amapá (UNIFAP).

${ }^{3}$ Biomedicale, Dottorato di Ricerca in Malattie Tropicali, Professore e ricercatore del Corso di Medicina dell'Università Federale di Amapá (UNIFAP).

${ }^{4}$ Medico dell'Università Federale di Amapá (UNIFAP).

${ }^{5}$ Medico dell'Università Federale di Amapá (UNIFAP).

${ }^{6}$ Università Federale di Amapá (UNIFAP).

${ }^{7}$ Biologo, Dottore di Ricerca in Malattie Topiche, Professore e ricercatore del Corso di Educazione Fisica dell'Università Federale di Pará (UFPA).

${ }^{8}$ Teologo, dottore in psicoanalisi clinica. Si è lavorato per 15 anni con la metodologia scientifica (metodo di creazione) nella guida di tutta la produzione scientifica dell'allievo del master e del docente. Specialista in ricerche di mercato e ricerche sulla salute.

${ }^{9}$ Dottore, professore e ricercatore del corso di medicina dell'Università federale di Amapá (UNIFAP).

${ }^{10}$ Dottore, professore e ricercatore del corso di medicina dell'Università federale di Amapá (UNIFAP).

${ }^{11}$ Dottore, Dottorato di Ricerca in Scienze Mediche, Professore e ricercatore del Corso di Medicina dell'Università Federale di Amapá (UNIFAP).

12 Medico, professore e ricercatore del corso di medicina dell'Università federale di Amapá (UNIFAP).
}

RC: 80368

Disponibile in: https://www.nucleodoconhecimento.com.br/saude/discentes-de- 


\section{ASTRATTO}

Le sostanze psicoattive, chiamate anche sostanze psicotrope, sono prodotti che hanno la capacità di promuovere stimoli o cambiamenti sensoriali, influenzando le emozioni e il livello di coscienza di chi le usa. Lo scopo di questo studio era quello di analizzare l'uso di sostanze psicoattive tra gli studenti di medicina dell'Università Federale di Amapá (UNIFAP) nel 2018. È stato condotto uno studio trasversale quantitativo e qualitativo, che ha utilizzato un campione di convenienza non probabilistica degli studenti universitari. In questa università, tra gli studenti di medicina, è stato notato che l'uso di farmaci psicotropi si verifica principalmente a causa dell'uso di alcol e analgesici. Nel contesto medico, a causa dell'accesso facilitato ai farmaci soggetti a prescrizione medica, il loro uso è molto più ampio in questo pubblico di professionisti e accademici, e l'uso di tali sostanze è talvolta nascosto e c'è una difficoltà a parlare e cercare aiuto.

Parole chiave: Studenti universitari, sostanza psicoattiva, medicina.

\section{INTRODUZIONE}

Le sostanze psicoattive, chiamate anche sostanze psicotrope, sono prodotti che hanno la capacità di promuovere stimoli o cambiamenti sensoriali, influenzando le emozioni e il livello di coscienza di chi le usa. II loro stimolo può essere diverso da persona a persona, poiché, sebbene organicamente simili, gli esseri umani possiedono caratteristiche individuali. Inoltre, i fattori che di solito sono determinanti per il grado di stimolazione sensoriale sono la quantità e il tipo di farmaci psicotropi, oltre al contesto in cui viene utilizzato (BRASIL, 2017).

In Brasile, oltre alle sostanze illecite (Anfetamine, marijuana, cocaina, crack, eroina e altre), c'è un ampio consumo di droghe lecite, come alcol, tabacco e analgesici (BRASIL, 2017). Molti ritengono che l'uso di questi farmaci sia quasi esclusivo del

RC: 80368

Disponibile in: https://www.nucleodoconhecimento.com.br/saude/discentes-de- 
pubblico minorile, ma erroneamente, poiché il tasso di anziani che li usano cronicamente è aumentato (DINIZ et al., 2017).

Tali sostanze possono causare vari tipi di reazioni nel sistema nervoso dell'individuo e possono essere depressori, causando una diminuzione delle attività del sistema nervoso, riducendo fattori importanti come la capacità di messa a fuoco, attenzione e ragionamento (alcol, tranquillanti, eroina e altri). Possono anche essere allucinogeni, alterando la realtà percepita dai sensi (marijuana, LSD e sostanze da prodotti naturali come funghi e piante). Gli stimolanti possono innescare cambiamenti nervosi in grado di generare momenti euforici estrapolati - oltre ad essere usati come risorsa per trattare problemi di attenzione e disturbi neurologici - e risvegliare l'individuo per un tempo completo. Esempi di queste sostanze sono cocaina, crack, tabacco, anfetamine e, ampiamente diffusa in tutto il mondo, caffeina (BRASIL, 2017).

L'alcol, sebbene sia una droga legale e comunemente usato nei social media, ha capacità psicotrope. II suo uso può indurre gli individui a presentare alterazioni comportamentali, e l'intossicazione delle donne da esso di solito si verifica più rapidamente, al fine di avere quantità più elevate di grasso, percentuali più basse di acqua e una minore produzione di alcol deidrogenasi (ADH), un enzima responsabile della degradazione dell'alcol nel corpo (SILVA et al., 2019). È caratterizzato come un fattore di rischio per una serie di lesioni, come quelle di ordine intenzionale violenza interpersonale, suicidio - e violenza involontaria, come ustioni, intossicazioni, annegamenti, tubercolosi, tumori, malattie del fegato, incidenti automobilistici e molti altri. Di tutto l'alcol consumato nel mondo, circa un quarto non è registrato, proveniente da fabbricazioni fatte a mano, adulterate o contraffatte (WHO, 2018). In Brasile, questo tasso è di circa il $15 \%$ e il pubblico che consuma bevande alcoliche più abusivamente è maschio, e la fascia di età più diffusa è di 1834 anni (ANDRADE, 2020).

Il fumo è caratterizzato dall'essere uno dei principali fattori di rischio per numerose malattie, tra cui i più vari tipi di tumori e malattie cardiopolmonari e la causa

RC: 80368

Disponibile in: https://www.nucleodoconhecimento.com.br/saude/discentes-de- 
prevenibile di morte. In tutto il mondo, più di 7 milioni di persone muoiono ogni anno a causa di problemi di salute causati dal fumo, e di questo totale, il Brasile ha circa 150 mila morti. Nel 2018, la percentuale di fumatori di età superiore o superiore a 18 anni era del 9,3\% nel paese. Nel settore sanitario, il Brasile spende quasi 40 miliardi di $R \$$ all'anno per malattie derivanti dal consumo di tabacco (FIGUEIREDO et al., 2017; BRASIL, 2019).

L'elevato consumo di analgesici in Brasile è fortemente legato all'altissimo tasso di prevalenza del dolore cronico (DC) (circa il 41\% della popolazione ha questo tipo di dolore). La causa principale del DC è di solito l'attività eseguita sul lavoro. Per il trattamento analgesico, i farmaci antinfiammatori non steroidei, aggiunti al dipirone e al paracetamolo, sono la linea di trattamento più diffusa. Tra gli analgesici, possiamo anche evidenziare la classe di oppiacei, utilizzati per trattare il dolore moderato e intenso, essendo ampiamente utilizzati nel trattamento del dolore dei pazienti oncologici (BARROS et al., 2019). In Brasile, nel 2015, è stato scoperto che circa 4,5 milioni di persone avevano già usato gli oppiacei in modo non prescritto ad un certo punto della loro vita (BASTOS et al., 2017).

Tra le droghe di abuso (droghe che non sono state adeguatamente prescritte da un professionista sanitario addestrato a farlo), come anfetamine, LSD, crack e cocaina, ad esempio, possiamo evidenziare la marijuana - Cannabis sativa - perché è stata utilizzata fin dall'inizio dell'umanità ed è la terza droga più consumata al mondo, con alcol e tabacco rispettivamente i più utilizzati (VANJURA et al. , 2018). Storicamente, nel XVIII secolo, molto prima di diventare una sostanza illecita nel paese, la marijuana è stata prodotta su larga scala in Brasile, con lo scopo di utilizzarla principalmente per la produzione di corde su navi portoghesi, generando profitto e favorendo la bilancia commerciale (PEREIRA et al., 2018). In Brasile, la marijuana è la droga illecita più consumata e circa il 7,7\% dei brasiliani tra i 12 e i 65 anni l'ha usata almeno una volta nella vita (BASTOS et al., 2017). Le sue proprietà comprendono abilità psicotrope e possono produrre allucinazioni ed effetti depressivi

$\mathrm{RC}: 80368$

Disponibile in: https://www.nucleodoconhecimento.com.br/saude/discentes-de- 
sul sistema nervoso. Inoltre, a volte è anche usato come trattamento per alcune patologie (VANJURA et al., 2018).

L'inizio del consumo psicoattivo di droghe di solito si verifica nella fascia di età di 12 e 24 anni. L'ingresso degli studenti nelle università di tutto il pianeta avviene di solito nella fascia di mezza età, un fattore che, poiché l'università rappresenta una fase di transizione e un ampio cambiamento nella vita dell'individuo, coincide con il più alto tasso di abuso psicotropo. Tra le sostanze utilizzate tra gli studenti universitari, alcol e tabacco sono confermati rispettivamente come i più utilizzati, e quindi concordano con lo standard di classificazione mondiale dell'uso psicotropo (OLASHORE et al., 2018). L'uso di donne psicoattive, e tali sostanze possono avere impatti negativi sulla vita personale e accademica di questi studenti, a causa dell'assenza dalle lezioni, ritardi e mancanza di attenzione (SANTOS et al., 2019). Tuttavia, i farmaci leciti, come l'alcol, possono essere fattori di rischio per questi studenti, poiché il comportamento e il giudizio degli individui possono essere alterati negativamente, portando ad atteggiamenti rischiosi, come il sesso non protetto (a volte culminato in una possibile contaminazione da virus dell'immunodeficienza acquisita - HIV - o gravidanza indesiderata, oltre ad altre possibili infezioni sessualmente trasmissibili) , violenza interpersonale e altri problemi (WHO, 2018).

Uno dei corsi con i posti vacanti più contestati in Brasile è la Medicina. Per avere la possibilità di entrare e diventare un accademico in questo settore, lo studente prevestibolare ha bisogno di una dedizione completa agli studi, al fine di costruire e tagliare le proprie conoscenze per il test vestibolare. Nel bel mezzo di questo arduo processo, molti studenti spesso sviluppano disturbi d'ansia, troppo stress e anche depressione. II requisito emotivo, legato alle cariche familiari (spesso presenti) per l'approvazione sono fattori che possono incitare tali disturbi e danneggiare la salute psicologica dello studente (SANTOS et al., 2017).

In considerazione del gran numero di fattori che causano stress nel corso di medicina, la cognizione e la psicologia degli studenti sono costantemente

$\mathrm{RC}: 80368$

Disponibile in: https://www.nucleodoconhecimento.com.br/saude/discentes-de- 
influenzate, oltre alla loro condizione fisiologica, innescando problemi psichiatrici organici e perdita della qualità della vita. Per comprendere la gravità di questi fattori, è necessario comprenderli, al fine di trovare l'origine dei problemi per risolverli (LIMA et al., 2016). Lo stress psicologico è solitamente associato a effetti accademici negativi, come difficoltà a dormire, uso di sostanze psicotrope, esaurimento, diminuzione dell'apprendimento (e conseguente impatto negativo sull'allenamento), mancanza di empatia con i pazienti e persino ideazione suicidale (MCLUCKIE et al., 2018). Tuttavia, al fine di ottenere una visione più completa dei problemi, è necessario osservare altri fattori coinvolti nello studente, come la possibilità di problemi finanziari, relazioni, mancanza di tempo, grande responsabilità, supporto $\mathrm{e}$ orientamento inadeguati nella loro formazione, frequente esposizione alla morte e alla sofferenza dei pazienti e altre circostanze complesse (HILL et al., 2018). Oltre a questi problemi, molti studenti di medicina finiscono per non cercare aiuto medico tutte le volte che dovrebbero, non riuscendo a risolvere i problemi che sorgono. In generale, solo l'8-15\% circa di questi studenti cerca un aiuto psichiatrico durante la laurea, anche se soffre costantemente a causa di tali problemi (VASCONCELOS et al., 2015).

\section{OBBIETTIVO}

Analizzare l'uso di sostanze psicoattive tra gli studenti di medicina dell'Università Federale di Amapá nel 2018.

\section{METODO}

Studio trasversale quantitativo e qualitativo, che utilizzava un campione di convenienza, non probabilistica, degli studenti universitari. Per la raccolta dei dati, il campione ha avuto 204 studenti di sei anni del corso di medicina dell'Università federale di Amapá (UNIFAP). La parte I dello strumento auto-applicabile convalidato in portoghese "(Drug Use Screening Inventory-Revised-DUSI-R) (DE MICHELI e FORMIGONI, 2002), è stata utilizzata, sviluppata per valutare e identificare l'abuso di

RC: 80368

Disponibile in: https://www.nucleodoconhecimento.com.br/saude/discentes-de- 
sostanze psicoattive, lecite e illecite e che misura l'uso di 13 sostanze psicoattive nell'ultimo mese. L'applicazione è avvenuta tramite modulo online e i dati ottenuti sono stati organizzati e tabulati in fogli di lavoro di programmi Excel, un componente della suite Office di Microsoft Corporation.

Il progetto è stato presentato e approvato dal Comitato Etico della Ricerca (CEP) (CAAE 89616818.2.0000.0003), secondo il Protocollo della Piattaforma Brasile, seguendo i principi etici e soddisfacendo le considerazioni etiche di cui alla risoluzione n. 466, del 12 dicembre 2012, del Consiglio Nazionale della Salute (CNS).

\section{RISULTATI}

L'analisi mostra che le principali sostanze utilizzate da tutte le classi erano alcol $(67,65 \%)$, analgesici $(49,51 \%)$ e tabacco $(9,80 \%)$. L'unica sostanza psicoattiva che viene consumata dalla maggior parte è l'alcol.

I dati mostrano l'uso di sostanze psicoattive negli ultimi trenta giorni da parte degli studenti di medicina tra il 2013 e il 2018, per genere e tipi di sostanze (Figura 1). II consumo di alcol e tabacco è più elevato negli studenti maschi (rispettivamente $36,27 \%$ e $7,37 \%)$, e l'uso analgesico è leggermente più elevato nelle femmine (25\%).

RC: 80368

Disponibile in: https://www.nucleodoconhecimento.com.br/saude/discentes-de- 
II grafico 1 Mostra l'uso di sostanze psicoattive negli ultimi trenta giorni da parte degli studenti di medicina tra il 2013 e 2018, per genere e tipi di sostanze.

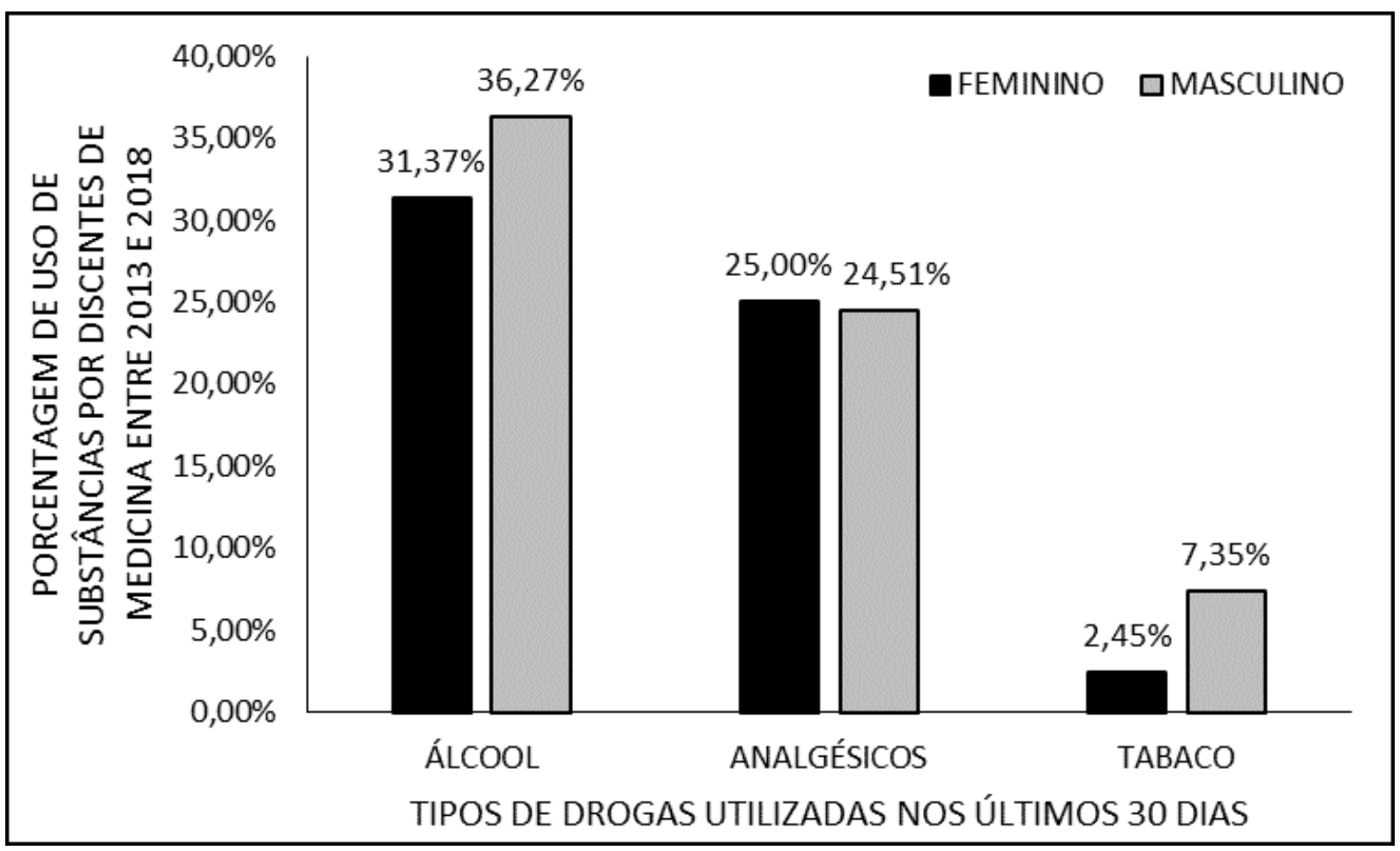

Fonte: Prodotto dagli autori con dati di ricerca.

Per quanto riguarda la percentuale e le tipologie di sostanze psicoattive utilizzate negli ultimi trenta giorni dagli studenti di medicina tra il 2013 e 2018, per genere (figura 2), negli uomini il numero è leggermente più alto (51\%) rispetto alle donne $(49 \%)$. Si noti la variazione annuale che dimostra questa quasi uguaglianza.

RC: 80368

Disponibile in: https://www.nucleodoconhecimento.com.br/saude/discentes-de- 
Figura 2 Mostra i tipi di sostanze psicoattive utilizzate negli ultimi trenta giorni dagli studenti di medicina tra il 2013 e il 2018, per genere.

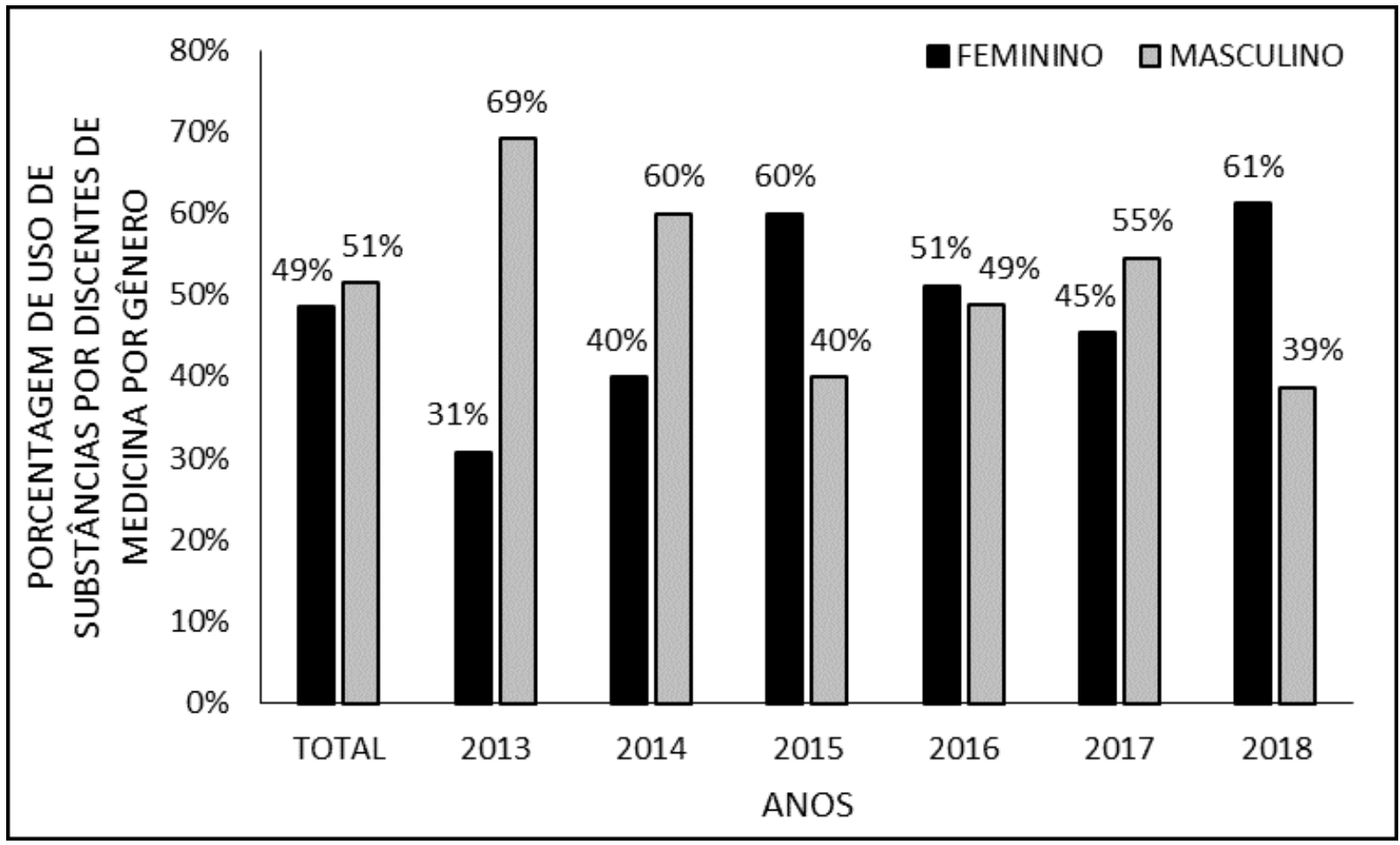

Fonte: Prodotto dagli autori con dati di ricerca.

La figura 3 mostra i tipi di sostanze psicoattive utilizzate negli ultimi trenta giorni dagli studenti di medicina tra il 2013 e il 2018, per anno e per genere. Nelle classi più anziane (2013 e 2014) non vi è alcun uso dichiarato delle sostanze descritte. Nella classe del 2015 c'è stato l'uso di ecstasy (0,49\%), marijuana $(0,49 \%)$, allucinogeni $(0,49 \%)$, tranquillanti $(0,49 \%)$ e altri $(0,49 \%)$, tutti per i maschi. Nella classe del 2016 i dati mostrano l'uso di tutte le sostanze descritte. Quest'anno il consumo di anfetamine (donne 0,98\% e uomini 0,49\%), ecstasy (donne 0,98\%), cocaina/crack (donne 0,49\%), marijuana (donne $1,47 \%$ e uomini 2,94\%), allucinogeni (donne 0,49\%), tranquillo (donne 1,47\% e uomini 0,49\%), oppiacei (donne 0,49\%), fenilciclina (donne 0,49\% e uomini 0,49\%), anabolizzanti (donne 0,49\%), inalanti / solventi (donne 0,49\%) e altri (donne 0,49\% e uomini 0,49\%). Nel 2017, consumo di anfetamine (donne 0,49\% e uomini 0,98\%), cocaina/crack (donne 0,49\% e uomini

$\mathrm{RC}: 80368$

Disponibile in: https://www.nucleodoconhecimento.com.br/saude/discentes-de- 
0,49\%), marijuana (donne $0,49 \%$ e uomini $0,98 \%$ ), allucinogeni (uomini $0,49 \%$ ), tranquillanti (donne $0,49 \%$ e uomini $0,98 \%$ ), oppiacei (donne $0,49 \%$ ), anabolizzanti (uomini $0,49 \%$ ) e altri (donne 0,49\%). Nel 2018 le sostanze psicoattive segnalate sono state anfetamine (donne 0,49\% e uomini 1,49\%), ecstasy (uomini 0,49\%), marijuana (donne $0,49 \%$ e uomini $1,47 \%$ ), allucinogeni (uomini $0,49 \%$ ) e tranquillanti (donne $0,49 \%$ e uomini $0,49 \%$ ).

RC: 80368

Disponibile in: https://www.nucleodoconhecimento.com.br/saude/discentes-de- 
Figura 3 Mostra i tipi di sostanze psicoattive utilizzate negli ultimi trenta giorni dagli studenti di medicina tra il 2013 e il 2018 , per anno e per genere.

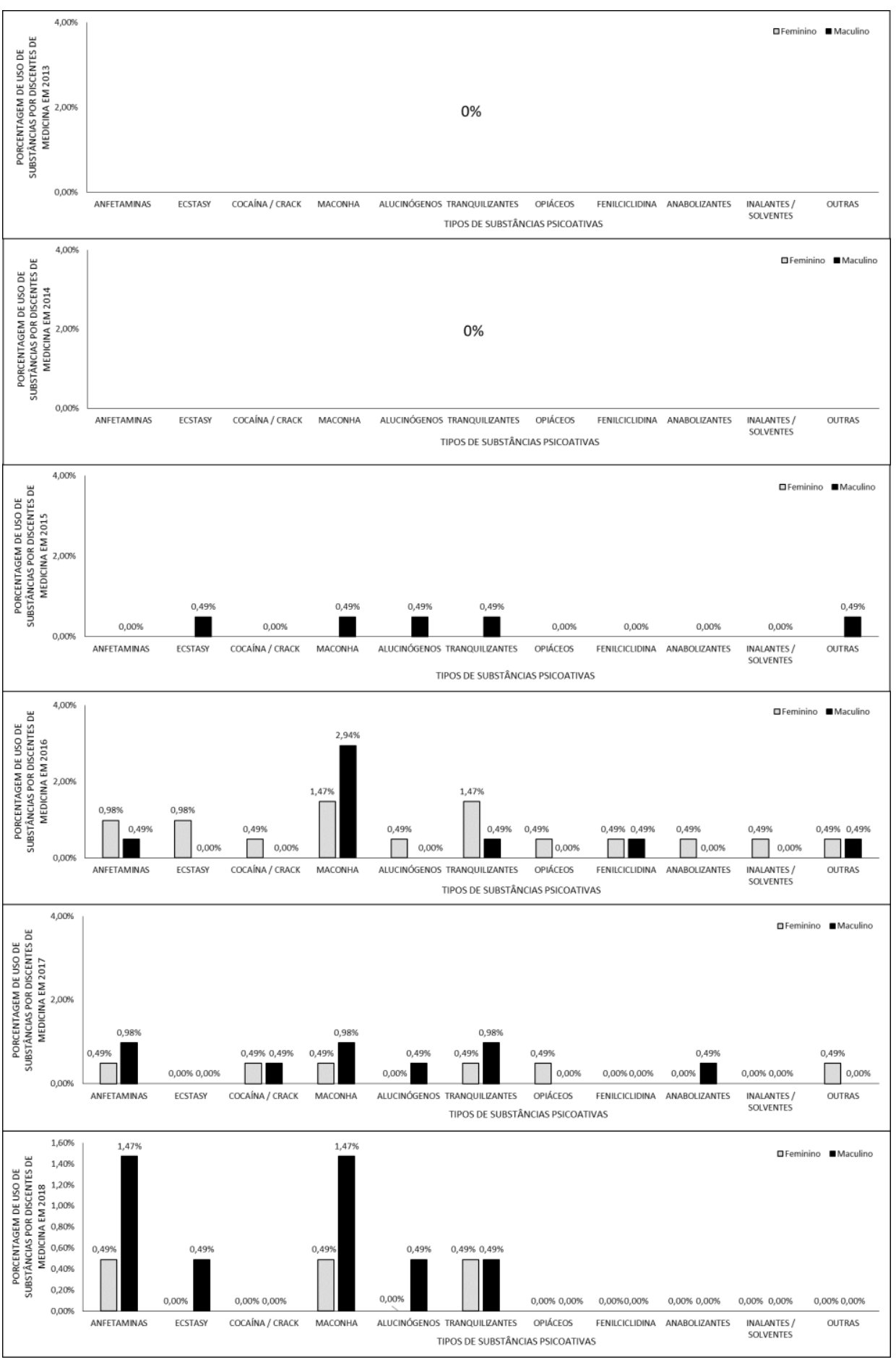

Fonte: Prodotto dagli autori con dati di ricerca.

RC: 80368

Disponibile in: https://www.nucleodoconhecimento.com.br/saude/discentes-de$\underline{\text { medicina }}$ 


\section{DISCUSSIONE}

In considerazione del brusco cambiamento di vita e dell'inserimento nell'ambiente sociale universitario, l'accademico attraversa un processo di trasformazione psicologica. Inoltre, altri fattori che contribuiscono a questa modifica organica e comportamentale derivano dallo stress quotidiano della graduazione. Aspetti come la vasta raccolta di responsabilità globali (che aumentano con il corso del corso), I'ansia, l'esaurimento, la distanza familiare (per gli accademici di altre località e anche per coloro che continuano a vivere con le loro famiglie, perché l'immensa quantità di contenuti da studiare finisce per privare i momenti di fraternizzazione e tempo libero) incorrono nella maggiore possibilità di utilizzare sostanze psicoattive, in particolare alcol (DELFINO et al., 2018). L'uso di farmaci psicotropi da parte di medici, studenti di medicina e operatori sanitari in generale è comune, poiché una parte considerevole di essi produce o ha già usato queste sostanze (TALIH et al., 2018).

Sebbene, secondo gli studi, le donne finiscano per iniziare l'uso di sostanze prima (con l'uso di marijuana, oppioidi, ansiolitici e altri), i maschi predominano in relazione all'uso cronico di alcol e tabacco e, di per sé, la voce "maschio" è legata come fattore di rischio per l'uso di sostanze psicoattive (FERNANDES et al. , 2017). Per quanto riguarda i fattori predisponenti all'uso di psicofarmaci, è in contatto che la partecipazione degli studenti nei centri accademici e il reddito superiore a 40 salari minimi (circa il 92\% degli studenti che si adattano a questo problema usano alcol, oltre a circa il 39\% fanno uso di droghe illecite) fanno più uso di tali sostanze (TRINDADE et al., 2018).

Negli studi effettuati con confronti tra alcune università nelle regioni sud-est e nordest, il modello di consumo delle principali sostanze psicotrope da parte degli studenti universitari era molto simile a livello di genere. Nel campione in questione, il consumo più elevato di alcol e marijuana si è verificato dai maschi, rispettivamente con il $74,5 \%$ e il $10,4 \%$, rispetto al $69,8 \%$ del consumo di alcol e al $7,9 \%$ della

RC: 80368

Disponibile in: https://www.nucleodoconhecimento.com.br/saude/discentes-de- 
marijuana femminile. (TRINDADE et al., 2018). Con il corso e il suo conseguente progresso di difficoltà e domanda, anche l'uso di sostanze psicoattive tende ad aumentare, poiché aumentano anche lo stress, l'ansia, l'esaurimento e molti altri fattori negativi, portando all'uso più pronunciato di questi

Già considerato come fattori di rischio per l'uso di psicofarmaci, il tasso di utilizzo di sostanze illegali è più elevato negli uomini (FERNANDES et al., 2017). Al fine di migliorare le loro prestazioni accademiche (tenendoli svegli, migliorando il loro umore e la loro memoria, oltre a migliorare la loro capacità di concentrazione e conseguente apprendimento) molti studenti di medicina usano farmaci come il ritalina (cloridrato metilfenidato) e le anfetamine, entrambi farmaci stimolanti del sistema nervoso. Tuttavia, sebbene si affidino ai benefici di questi farmaci per migliorare le loro prestazioni, ci sono numerose conseguenze negative di questo uso, come l'aumento della pressione sanguigna a livelli pericolosi, l'euforia, l'aquipnea (aumento del ritmo respiratorio) e altre implicazioni organiche. In uno studio condotto presso la Babol University of Medical Sciences, è stato riscontrato che del campione di 49 studenti di medicina che hanno fatto uso di farmaci stimolanti, 29 dei quali erano maschi, per un totale di circa il $59,2 \%$ del valore del campione (FALLAH et al., 2018).

L'uso di sostanze psicoattive tra professionisti medici e studenti di medicina è completo e questo uso può essere legato a una domanda educativa e professionale estrema. In considerazione dell'ampio accesso ai farmaci più vari disponibili, vi è un alto tasso di utilizzo di sostanze prescritte da medici e studenti di medicina, poiché l'esaurimento fisico e la capacità di dipendenza da questi farmaci culminano nel loro ampio uso. Inoltre, sebbene esistano tecniche in grado di monitorare l'uso di tali farmaci, vi è una grande difficoltà nell'ammettere il loro uso e nel riferire che un collega attraversa questa situazione. Pertanto, l'uso generale di queste sostanze avviene in generale (DUMITRASCU et al., 2014).

RC: 80368

Disponibile in: https://www.nucleodoconhecimento.com.br/saude/discentes-de- 


\section{CONCLUSIONI}

In considerazione del cambiamento dell'ambiente sociale, entrando nell'università, l'accademico subisce una serie di trasformazioni psichiche, che sono influenzate dall'ambiente accademico e dalle interazioni interpersonali da lui fornite. In questo articolo, il consumo di alcol e alcune sostanze psicoattive, come la marijuana, possono essere incitate.

A livello nazionale, gli studenti pre-vestiboli affrontano grandi sfide per accedere al corso di medicina, poiché i loro posti vacanti sono molto contestati ed è necessaria una grande dedizione agli studi per poter essere approvati. A causa di tali difficoltà, molti finiscono per sviluppare ansia, stress e disturbi psichici.

A causa delle numerose richieste e responsabilità del corso di medicina, diversi fattori negativi finiscono per essere innescati nella vita dello studente, come ansia, stress, esaurimento, difficoltà a dormire e altri problemi organici.

Al fine di prolungare le ore di studio e concentrazione nelle attività mediche, molti accademici utilizzano sostanze psicoattive illecite per aumentare le loro prestazioni, tuttavia, possono ottenere numerose conseguenze fisiologiche negative a causa di ciò.

Presso l'Università Federale di Amapá, tra gli studenti di medicina, è stato notato che l'uso di psicofarmaci si verifica principalmente a causa dell'uso di alcol e analgesici. Tuttavia, anche se in una piccola parte del campione, vi era anche l'uso di altre sostanze, come tranquillanti, tabacco, ecstasy, cocaina, crack e altre.

La predominanza dell'uso di alcol e tabacco è maschile, corrispondente ai parametri mondiali, poiché l'itesite "maschio" è considerato un fattore di rischio per l'uso di sostanze psicoattive. Tuttavia, si noti che l'uso di analgesici è più alto tra le femmine

RC: 80368

Disponibile in: https://www.nucleodoconhecimento.com.br/saude/discentes-de- 
e che, come per altre sostanze - in particolare la marijuana - il tasso di utilizzo è simile.

Nel contesto medico, a causa dell'accesso facilitato ai farmaci soggetti a prescrizione medica, il loro uso è molto più ampio in questo pubblico di professionisti e accademici, e l'uso di tali sostanze è talvolta nascosto e c'è una difficoltà a parlare e cercare aiuto.

\section{RIFERIMENTI}

ANDRADE, A. G. D. Álcool e a Saúde dos Brasileiros: Panorama 2020. São Paulo: 2020. 152p. Disponível em: < https://cisa.org.br/images/upload/Panorama_Alcool_Saude_CISA2020.pdf >.

BARROS, G. A. M. D. et al. Uso de analgésicos e o risco da automedicação em amostra de população urbana: estudo transversal. Rev Bras Anestesiol., v. 69, n. 6, p. 529-536, 2019.

BASTOS, F. I. P. M. et al. III Levantamento Nacional sobre o uso de drogas pela população brasileira. Rio de janeiro RJ: ICICT/Fiocruz, 2017. 528p. Disponível em:

https://www.arca.fiocruz.br/bitstream/icict/34614/1/II\%20LNUD_PORTUGU\%c3\%8a S.pdf $>$.

BRASIL. Substâncias psicoativas e seus efeitos. Brasilia DF: SENAD: 24 p. 2017. Vigilância de fatores de risco e proteção para doenças crônicas por inquérito telefônico. Brasilia DF: Ministério da Saúde: 131 p. 2019.

DE MICHELI, D.; FORMIGONI, M. L. O. S. Psycometric properties of the Brazilian version of DUSI (Drug Use Screening Inventory). Alcoholism: Clinical and Experimental Research, v. 26, p. 1523-1528, 2002.

$\mathrm{RC}: 80368$

Disponibile in: https://www.nucleodoconhecimento.com.br/saude/discentes-de- 
DELFINO, N. H. et al. Expectativas e perfil do uso de álcool em acadêmicos de medicina. Arq. Catarin Med., v. 47, n. 4, p. 132-145, 2018.

DINIZ, A. et al. Uso de substâncias psicoativas em idosos: uma revisão integrativa. Revista Psicologia: Teoria e Prática, v. 19, n. 2, p. 23-41, 2017.

DUMITRASCU, C. I. et al. Substance use among physicians and medical students. Medical Student Research Journal, v. 3, p. 26-35, 2014.

FALLAH, G. et al. Stimulant use in medical students and residents requires more careful attention. Caspian J Intern Med, v. 9, n. 1, p. 87-91, 2018.

FERNANDES, T. F. et al. Uso de susbstâncias psicoativas entre universitários brasileiros: perfil epidemiológico, contextos de uso e limitações metodológicas dos estudos. Cad. Saúde Colet., v. 25, n. 2, p. 498-507, 2017.

FIGUEIREDO, V. C.; TURCI, S. R. B.; CAMACHO, L. A. B. Controle do tabaco no Brasil: avanços e desafios de uma política bem sucedida. Cad. Saúde Pública, v. 33, p. 54-56, 2017.

HILL, M. R.; GOICOCHEA, S.; MERLO, L. J. In their on words: stressors facing medical students in the millenial generation. Medical Education Online, v. 33, p. 110, 2018.

LIMA, R. L. D. et al. Estresse do estudante de medicina e rendimento acadêmico. Revista Brasileira De Educação Médica, v. 40, n. 4, p. 678-684, 2016.

MCLUCKIE, A. et al. The relationship between psychological distress and perception of emotional support in medical students and residents and implications for educational institutions. Acad. Psychiatry, v. 42, n. 1, p. 41-47, 2018.

RC: 80368

Disponibile in: https://www.nucleodoconhecimento.com.br/saude/discentes-de- 
OLASHORE, A. A. et al. Psychoactive substance use among firstyear students in a Botswana university: pattern and demographic correlates. BMC Psychiatry, v. 18, n. 270, p. 1-9, 2018.

PEREIRA, J. R. et al. Cannabis Sativa: aspectos relacionados ao consumo de maconha no contexto brasileiro. RAHIS, v. 15, n. 1, p. 1-16, 2018.

SANTOS, D. D. M. et al. Use of psychoactive substances among university students. Revista Eletrônica Saúde Mental Álcool Drog, v. 15, n. 3, p. 1-9, 2019.

SANTOS, F. S. et al. Estresse em estudantes de cursos preparatórios e de graduação em medicina. Revista Brasileira De Educação Médica, v. 41, n. 2, p. 194-200, 2017.

SILVA, M. D. G. B. D.; LYRA, T. M.; DINIZ, G. T. O padrão de consumo de álcool entre as usuárias das unidades de saúde da família no município do Recife (PE). Saúde Debate, v. 42, n. 122, p. 836-847, 2019.

TALIH, F. et al. Examining burnout, depression, and attitudes regarding drug use among lebanese medical students during the 4 years of medical school. Academic Psychiatry, v. 42, n. 2, p. 288-296, 2018.

TRINDADE, B. P. D. A.; DINIZ, A. V.; JÚNIOR, A. R. S.-. Uso de drogas entre estudantes universitários: uma perspectiva nacional. Revista de Medicina e Saúde de Brasília, v. 7, n. 1, p. 52-60, 2018.

VANJURA, M. D. O. et al. Drogas de abuso: maconha e suas consequências. Rev Cient FAEMA, v. 9, p. 565-569, 2018.

VASCONCELOS, T. C. D. et al. Prevalência de sintomas de ansiedade e depressão em estudantes de medicina. 39, n. 1, p. 135-142, 2015.

$\mathrm{RC}: 80368$

Disponibile in: https://www.nucleodoconhecimento.com.br/saude/discentes-de- 
WHO. Global status report on alcohol and health 2018. WHO, 2018. 472p. Disponível em:

https://apps.who.int/iris/bitstream/handle/10665/274603/9789241565639-eng.pdf > .

Inviato: Maggio 2020.

Approvato: Maggio 2020.

RC: 80368

Disponibile in: https://www.nucleodoconhecimento.com.br/saude/discentes-demedicina 